\title{
Double blind placebo controlled trial of pizotifen syrup in the treatment of abdominal migraine
}

\author{
David N K Symon, George Russell
}

\begin{abstract}
Fourteen children with abdominal migraine were treated with pizotifen and placebo in a double blind crossover trial. The results showed pizotifen to be clearly superior to placebo in the prophylaxis of abdominal migraine. The importance of clearly distinguishing abdominal migraine from other forms of recurrent abdominal pain is emphasised. (Arch Dis Child 1995; 72: 48-50)
\end{abstract}

Keywords: abdominal pain, migraine, pizotifen.

It has long been recognised that migraine is not just a syndrome of headache, but that the headache is often associated with abdominal symptoms. Abdominal pain probably related to migraine but occurring in the absence of headache was described in adults by Buchanan in 1921, 1 although the term 'abdominal migraine' was not introduced until the next year. $^{2}$

Similar symptoms of episodic pyrexia, headache, vomiting, and abdominal pains in childhood were described by Wyllie and Schlesinger, who called these 'periodic disorders of childhood' and who noted that the symptoms might persist as vomiting with or without migraine in adult life. ${ }^{3}$ Cullen and Macdonald described the syndrome further and noted the frequency of migraine in adult relatives of children with periodic syndrome. ${ }^{4}$ The study of abdominal migraine was hindered for many years by the absence of a clear definition of the syndrome and we attempted to rectify this in an earlier paper. ${ }^{5}$ Epidemiological studies using this definition have shown abdominal migraine to be a common disorder present in $1 \cdot 7-2 \cdot 7 \%$ of schoolchildren. ${ }^{6} 7$

Objective evidence of the relation between abdominal migraine and migraine headaches has now become available. Children with abdominal migraine have been shown to have abnormalities of fast wave activity in the visual evoked response similar to children with migraine headaches. ${ }^{8} 9$ Despite this evidence, the existence of abdominal migraine is not yet universally accepted. ${ }^{10} 11$

Pizotifen has been shown to be effective in migraine prophylaxis in several double blind studies in adults. There have been few studies in children, so clinical practice has been based largely on the results of experience and clinical trials in adults. ${ }^{12} \mathrm{~A}$ double blind study of 39 children with migraine headaches given pizotifen $0.5 \mathrm{mg}$ twice or three times daily found no significant difference between pizotifen and placebo with respect to the frequency, duration, and severity of attacks. ${ }^{13}$ Despite this, the drug is widely used for migraine prophylaxis in children. In our clinical practice we have found pizotifen to provide effective prophylaxis in $70 \%$ of children with migraine. ${ }^{14}$

The purpose of this study is to evaluate pizotifen as a prophylactic drug in children with abdominal migraine.

\section{Patients and methods}

We studied 16 children aged 5-13 years attending a hospital general paediatric clinic. All children presented with a history of recurrent discrete episodes of abdominal pain fitting our previous definition of abdominal migraine. ${ }^{5}$

Entry criteria for the trial were recurrent attacks of central abdominal pain without radiation and with no features to suggest structural disease. Although the abdominal pain could be accompanied by headache, nausea, or vomiting, abdominal pain was the principal symptom in each patient. The abdominal pain had to be accompanied by facial pallor. Symptoms had been present for at least six months before the start of the study. Attacks were occurring at least twice monthly and each attack lasted for at least two hours. Patients were only accepted for the trial if one first degree relative or two second degree relatives had migraine or recurrent throbbing headaches.

All patients had a haematological profile, erythrocyte sedimentation rate, biochemical screen, and urine culture performed with normal results before entry to the trial.

After selection for the study patients were weighed and issued with a daily diary card for the child or parent to complete, giving details of compliance, pain severity and duration, any other drugs taken, and details of any side effects observed. The patients were prescribed pizotifen syrup containing $0.25 \mathrm{mg}$ pizotifen/ $5 \mathrm{ml}$ or an identical placebo syrup. The two syrups were sugar free. The initial dose of syrup was $5 \mathrm{ml}$ twice daily.

After one month there was an optional return visit when any problems could be discussed with the child or parents. If the child did not return to the clinic contact was made by telephone. If there had been no improvement, whether or not the child had attended the clinic, the dose of syrup was increased to $5 \mathrm{ml}$ three times daily. 
Results in 14 children who completed the trial

\begin{tabular}{lcccccc}
\hline & $\begin{array}{c}\text { Placebo } \\
\text { (mean) }\end{array}$ & $\begin{array}{c}\text { Pizotifen } \\
\text { (mean) }\end{array}$ & $\begin{array}{l}\text { Mean (SEM) } \\
\text { difference }\end{array}$ & $\begin{array}{l}t \\
\text { Value }\end{array}$ & df & $\stackrel{p}{\text { Value }}$ \\
\hline Days of abdominal pain noted & $12 \cdot 50$ & $4 \cdot 29$ & $8 \cdot 21(2 \cdot 44)$ & 3.37 & 13 & 0.005 \\
Index of severity & 23.50 & $7 \cdot 29$ & $16 \cdot 2143(4 \cdot 77)$ & 3.4 & 13 & 0.005 \\
Index of misery & 81.50 & $25 \cdot 43$ & $56.07(17 \cdot 59)$ & 3.19 & 13 & 0.007 \\
\hline
\end{tabular}

At the end of the second month the patients were weighed and a further blood sample taken for haematological and biochemical screening. The diary card was collected and a replacement issued. A further supply of the different trial syrup was prescribed and the initial dose was again $5 \mathrm{ml}$ twice daily.

After a further month there was another optional return visit identical to that previously described.

At the final visit at the end of the fourth month the child was again weighed and a further blood sample obtained for haematological and biochemical screening. At this visit patients were asked which of the two treatment periods was the most effective.

The effect of the drug on the severity of attacks was assessed using two indices. The index of severity was calculated by allocating one mark for an attack graded as mild by the parent, two for an attack graded as moderate, and three for a severe attack, and totalling these for the duration of the trial treatment. The index of total misery was calculated on the basis that a prolonged mild attack might be equated with a shorter but more severe attack. An index was calculated based on the product of the severity index and the duration of attack, and totalled over the duration of the trial treatment.

Statistical analysis was by Student's $t$ test for pairs.

The syrup preparations for the trial were supplied by Sandoz Products Ltd, but the protocol was designed independently from the company and conducted and analysed independently by the authors.

\section{Results}

Fourteen children completed the trial. Two children were excluded from the analysis. One was issued with the trial drug and documentation but then did not return to the clinic for review. The second child did not keep a record of symptoms and analysis was impossible.

From the 14 patients analysed, 11 patients or their parents preferred pizotifen, two preferred placebo, and one was undecided.

During the period when receiving pizotifen, the patients had significantly fewer symptoms, whether the results were analysed in terms of the number of days on which abdominal pain was noted, or in terms of the indices of severity or misery which we calculated from the diary cards (table).

Only two patients reported side effects. One child had slight drowsiness during the first few days of receiving active treatment. This side effect had disappeared by the end of the first week. One patient had an increased appetite while receiving the placebo. This was also a transient phenomenon. The mean weight gain during the period of receiving pizotifen was $1.25 \mathrm{~kg}$ compared with $0.38 \mathrm{~kg}$ during the placebo period $(p=0.039)$. No biochemical nor haematological change was noted during the study.

\section{Discussion}

This study shows that pizotifen was clearly superior to placebo in the prophylaxis of childhood abdominal migraine. We had originally planned to study a larger group of children, but by the time our initial supply of drugs had reached its expiry date, it was obvious that there was a strong patient/parent preference for pizotifen compared with placebo. We therefore analysed the data already accumulated. The beneficial effects of pizotifen were so striking clinically, and the trial data so convincing statistically, that we felt it would be unethical to study further patients with a protocol involving the administration of placebo.

The results of this trial confirm the subjective impression gained from clinical practice in which we have observed that pizotifen is an effective prophylactic drug in about $70 \%$ of children with abdominal migraine. ${ }^{14}$ Continued benefit has been reported after stopping pizotife ${ }^{116}$ but in this study there was no evidence that those patients who received pizotifen first had a better response to placebo than those in whom the placebo was given first. It is possible that the duration of active treatment in this trial was too short to produce this effect; the patients reported previously ${ }^{15}$ had been receiving pizotifen for six months before treatment was stopped.

The importance of this study is that it confirms the efficacy of a treatment which is already widely used, and also that it supports the view that it is important to distinguish abdominal migraine, for which effective treatment is now available, as a diagnostic entity distinct from other categories of recurrent abdominal pain. We emphasise that children recruited for the trial fulfilled previously published diagnostic criteria for abdominal migraine ${ }^{5}$ and, in addition, had a family history of migraine or throbbing headache in either a first degree relative or two second degree relatives. There is no reason to believe that pizotifen would be effective in patients with abdominal pain who did not fulfil our diagnostic criteria, and it is unlikely to be useful in non-specific or psychogenic abdominal pain.

1 Buchanan JA. The abdominal crises of migraine. $f$ Ner Ment Dis 1921; 54: 406-12.

Brams WA. Abdominal migraine. $f A M A$ 1922; 78: 26-7

3 Wyllie WG, Schlesinger B. The periodic group of disorders in childhood. British fournal of Children's Diseases 1933; 30: $1-21$.

4 Cullen KJ, Macdonald WB. The periodic syndrome; its nature and prevalence. Med $\mathcal{F}$ Aust 1963; ii: 167-73.

5 Symon DNK, Russell G. Abdominal migraine: a childhood syndrome , Russell G. Abdominal migraine: a

6 Mortimer MJ, Kay J, Jaron A. Clinical epidemiology of childhood abdominal migraine in an urban general practice. Dev Med Child Neurol 1993; 35: 243-8. 
7 Abu-Arafeh IA, Russell G. Current controversies - abdominal migraine. Cephalalgia 1993; 13: 138-9.

8 Mortimer MJ, Good PA, Marsters JB, Addy DP. Visual evoked responses in children with migraine: a diagnostic test. Lancet 1990; 335: 75-7.

9 Mortimer MJ, Good PA. The VER as a diagnostic marker for childhood abdominal migraine. Headache 1990; 30 $642-5$

10 Symon DNK. Is there a place for 'abdominal migraine' as separate entity in the IHS classification? Yes! Cephalalgia 1992; 12: 345-6.

11 Hockaday JM. Is there a place for 'abdominal migraine' as a separate entity in the IHS classification? No! Cephalalgia 1992; 12: 346-8.

12 Symon DNK. Pizotifen. In: Gallai V, Guidetti V, eds. fuvenile headache. Etiopathogenesis, clinical diagnosis and therapy. Amsterdam: Elsevier Science, 1991: 405-8.

13 Gillies D, Sills M, Forsythe I. Pizotifen (Sanomigran) in childhood migraine. Eur Neurol 1986; 25: 32-5.

14 Symon DNK, Russell G. The general paediatrician's view of migraine - a review of 250 cases. In: Lanzi G, Balottin $\mathrm{U}$, Cernibori A, eds. Headaches in children and adolescents. U, Cernibori A, eds. Headaches in children

15 Symon DNK, Russell G. Continued benefit after stoppin pizotifen therapy in childhood migraine. Cephalalgi 1989; 9 (suppl 10): 422-3.

16 Peet KMS, Gray JA. Pizotifen (Sanomigran) in the prophylaxis of migraine headache: a long-term follow-up study. Cephalalgia 1989; 9 (suppl 10): 420-1.

\section{Commentary}

I agree with the authors that this is an important study. It is always comforting to learn that a widely used treatment has a rational basis.

Until recently, the migrainous nature of recurrent paroxysmal abdominal pain with facial pallor and a family history of migrainous headache has been contentious. The recent demonstration of the same fast wave abnormality in the visually evoked response of children with paroxysmal abdominal symptoms, ${ }^{1}$ as in those with migrainous headache, has helped to settle the debate. The present observation that the serotonin receptor stimulator, pizotifen, a proved treatment for cranial migraine, is equally effective in abdominal migraine is consistent with such a link.

The study also has important therapeutic implications. It is important to remember that prophylaxis is not appropriate in those children with mild, brief, or infrequent episodes. Pizotifen is also expensive.

Despite several convincing demonstrations to the contrary, the belief that recurrent abdominal pain without an organic basis is almost invariably psychogenic, dies hard. Hopefully, this paper, with others implicating defects in gastrointestinal motility for example, ${ }^{2}$ will be further nails in the coffin of that particular hypothesis.

I W BOOTH Institute of Child Health Nuffield Building, Francis Road, Birmingham B16 8ET

1 Mortimer MJ, Good PA, Marsters JB, Addy DP. Visual evoked responses in children with migraine: a diagnostic test. Lancet 1990; 335: 75-7.

2 Pineiro-Carrero VM, Andres JM, Davis RH, Mathias JR. Abnormal gastroduodenal motility in children and adolescents with recurrent functional abdominal pain. $\mathcal{F}$ Pediatr cents with recurrent 